\title{
Le revenu inconditionnel dans et par les discours : analyse lexicométrique et essai de typologie
}

Discourses on unconditional income, towards unconditional income with discourses: lexicometric analysis and proposed typology

El ingreso básico incondicional a través de los discursos: análisis lexicométrico e intento de tipología

\section{Cédric Leterme}

\section{OpenEdition}

\section{Journals}

Édition électronique

URL : https://journals.openedition.org/mots/23452

DOI : $10.4000 /$ mots. 23452

ISSN : 1960-6001

Éditeur

ENS Éditions

\section{Édition imprimée}

Date de publication : 5 juillet 2018

Pagination : 109-129

ISSN : 0243-6450

\section{Référence électronique}

Cédric Leterme, "Le revenu inconditionnel dans et par les discours : analyse lexicométrique et essai de typologie », Mots. Les langages du politique [En ligne], 117 | 2018, mis en ligne le 05 juillet 2020, consulté le 22 avril 2022. URL : http://journals.openedition.org/mots/23452 ; DOI : https://doi.org/ $10.4000 /$ mots. 23452 


\section{Le revenu inconditionnel dans et par les discours : analyse lexicométrique et essai de typologie}

Le débat qui se déroule aujourd'hui en Europe autour du versement d'une forme de "revenu inconditionnel ${ }^{1}$ connaît un succès croissant depuis quelques années, notamment à la faveur des conséquences de la crise économique et financière de 2008 ou encore de l'accélération de la «révolution numérique ». L'idée est désormais portée par des acteurs politiques de premier plan - comme le candidat socialiste à l'élection présidentielle française de 2017, Benoît Hamon -, tandis que des expérimentations concrètes se multiplient en Finlande, aux Pays-Bas ou encore en Italie.

Sous cette même idée coexistent cependant des projets très dissemblables, ce qui n'empêche pas bon nombre de partisans (et d'opposants!) de traiter ces propositions comme largement équivalentes, essentiellement pour des raisons stratégiques de diffusion large du thème (ou de condamnation en bloc). Notre objectif est de mettre à jour ces différences afin que les enjeux politiques qu'elles expriment puissent être clairement identifiés. Pour ce faire, notre travail se focalisera sur une analyse des discours, étant entendu que la façon dont ces différentes propositions se construisent et se légitiment dans et à travers le recours à un lexique particulier, en tension et en concurrence, nous informe sur le cadrage idéologique mobilisé.

Nous avons ainsi pratiqué une analyse lexicométrique sur un corpus de neuf textes proposant et défendant chacun une forme particulière de revenu inconditionnel. Six d'entre eux sont extraits d'auditions réalisées dans le cadre d'une mission d'information du Sénat français consacrée à la question en 2016. Les trois autres incarnent des courants qui n'étaient pas représentés dans le cadre de ces auditions. Ils ont été publiés dans des revues ayant consacré un dossier à cet enjeu. À travers l'analyse de ces différents textes, nous verrons non seulement à quel point il existe des différences politiques fondamentales entre

1. Nous utiliserons ce terme par convention pour désigner toutes les propositions de revenu versé à tous les membres d'une société sans contrepartie, notamment en termes d'activité. 
certaines des propositions de revenu inconditionnel les plus débattues, mais aussi comment ces différences politiques se construisent dans et à travers les discours qui les portent.

Notre article commence toutefois par un retour sur les raisons qui expliquent la popularité croissante de l'idée de revenu inconditionnel en Europe depuis quelques années, ainsi que sur les enjeux que cette popularité soulève. Nous exposerons ensuite les caractéristiques du corpus de textes retenu pour l'analyse, ainsi que les outils et avantages de la méthode lexicométrique. Nous terminerons enfin par une présentation des résultats issus de ce type d'analyse, complétée par une démarche de nature plus qualitative. Nous verrons que ces deux approches permettent à la fois d'identifier une typologie des propositions de revenu inconditionnel en fonction de leur rapport à l'économie capitaliste, et de montrer comment ces rapports se construisent concrètement dans et à travers le choix de certains mots, récits, arguments ou encore procédés rhétoriques.

\section{Le revenu inconditionnel comme objet de débat croissant depuis les années 2010}

L'idée d'attribuer à chaque membre d'une société donnée un revenu inconditionnel est une idée ancienne dont on peut faire remonter l'origine au moins jusqu'au XVIII' siècle (Bresson, 2007 ; Suplicy, 2006 ; Vanderborght, Van Parijs, 2005). Elle est toutefois restée longtemps marginale face aux développements progressifs de l'État social et des sociétés de plein-emploi. Il faudra d'ailleurs attendre leurs crises conjuguées dans le courant des années 1960-70 pour que le revenu inconditionnel s'invite réellement dans le débat public, d'abord par le biais de la nouvelle droite néolibérale (Friedman, 1962), puis d'une certaine gauche libertaire et/ou écologiste (Collectif Charles Fourier, 1985; Gorz, 2002), elle-même suivie par des mouvements de chômeurs et de précaires des années 1990 (Baudouin et al., 1989) ou encore par les théoriciens du «capitalisme cognitif» au début des années 2000 (Moulier-Boutang, 2007).

Durant toute cette période, le débat sur le revenu inconditionnel reste toutefois confiné à des cercles intellectuels et militants relativement restreints. Or, la situation change à partir des années 2010, et ce sous l'effet de plusieurs facteurs. On peut d'abord citer les conséquences de la crise économique et financière de 2008, à commencer par un chômage et/ou un sousemploi massif (OIT, 2017), ainsi qu'une stagnation économique que des économistes comme Robert J. Gordon, Paul Krugman ou encore Larry Summers n’hésitent pas à considérer comme «séculaire». Dans ces conditions, en effet, le mythe déjà bien entamé d'un retour possible au plein-emploi des «Trente Glorieuses » apparaît plus illusoire que jamais, et avec lui la possibilité de continuer à prendre l'emploi comme source principale (voire unique) de revenu et de droits sociaux. 
Or, ce sentiment est encore aggravé par l'accélération et l'approfondissement de la « révolution numérique $»^{2}$ qui bouleverse radicalement les relations de travail tout en faisant massivement disparaître des emplois (Colin, Verdier, 2012). D’un côté, en effet, «l'ubérisation» de l'économie pose la question de l'avenir même de la relation d'emploi en remplaçant celle-ci par de l'autoentrepreneuriat souvent dénué de protection et de stabilité (Babinet, 2016). De l'autre, les progrès récents de l'automatisation ne menacent plus seulement la majorité des emplois sous-qualifiés, mais de plus en plus d'emplois jusqu'ici considérés comme irremplaçables (Frey, Osborne, 2013). Face à ces évolutions, l'idée d'un revenu inconditionnel apparaît donc comme un remède à la disparition comme à la précarisation des emplois qui découlent de la « révolution numérique ». Elle peut également se revendiquer d'une logique de simplification administrative du fonctionnement de la protection sociale qui rencontre un écho particulier au moins depuis les années 1980 avec la mise en cause de la bureaucratisation excessive de l’État social.

Un troisième facteur est de nature environnementale, avec la prise de conscience croissante de l'urgence climatique, et plus largement de la nécessité de protéger notre écosystème, qui pousse de plus en plus d’individus à s’interroger sur les alternatives possibles à la société de croissance et au productivisme qui l'accompagne 3 . Or, de ce point de vue, un revenu inconditionnel peut être considéré comme un moyen efficace de ralentir la production de biens et de services potentiellement néfastes pour l'environnement, mais aussi de valoriser en parallèle des activités socialement et/ou écologiquement utiles qui peinent à se développer dans le cadre du fonctionnement économique capitaliste. Une autre tendance qui pourrait également expliquer en partie l'engouement récent pour le revenu inconditionnel s'inscrit dans la perte de centralité du rapport social «travail» et/ou de sa modification profonde qui s'observe en particulier dans les sociétés à économies «développées» (Méda, 2010).

Enfin, il faut également souligner l'intense travail de sensibilisation et de lobbying effectué par des partisans du revenu inconditionnel ces dernières années, dont les réseaux et les structures, à commencer par ceux du Basic Income Earth Network (BIEN), ont permis d'en maximiser les échos et les retombées, en particulier en Europe. La campagne européenne lancée en 2013 dans le cadre du nouveau «droit d'initiative citoyenne européenne » a ainsi été l'occasion de populariser l'idée à l'échelle du continent avec tout de même un résultat de près de 300000 signatures obtenues dans la quinzaine de pays

2. Le terme renvoie à l'idée d'une quatrième révolution industrielle liée à l'essor de l'intelligence artificielle, de l'automatisation ou encore du «big data», mais sa nature et sa portée exactes font encore l'objet de nombreux débats.

3. Le succès du film Demain (2015) et des initiatives qu'il met en avant en est une preuve parmi d'autres. 
mobilisés. Or, elle s'est également accompagnée de nombreuses initiatives nationales similaires (en Espagne, en Italie ou encore en Suisse par exemple) ayant à chaque fois permis de réintroduire la question au centre de l'actualité.

Dans ce contexte, on comprend mieux l'engouement politique et médiatique croissant que suscitent les propositions de revenu inconditionnel, qui sont tout de même passées en l'espace de quelques années du statut de position ultra-minoritaire à celui de propositions méritant d'être débattues dans des cénacles reconnus. C'est ainsi que des acteurs et des forces politiques de premier plan couvrant la quasi-totalité du spectre politique n'hésitent plus à les inscrire dans leur programme ou à en débattre4, tandis que des expérimentations se multiplient en Europe, notamment en Finlande, en Italie ou encore aux Pays-Bas, et que d'autres sont prévues ou sérieusement envisagées (en Écosse ou en France, par exemple).

Ce succès s'est toutefois très largement construit malgré (et en partie grâce à) une confusion entre les modalités et surtout les finalités poursuivies par les différentes propositions existantes. Car au-delà du principe minimal commun qui consiste à verser à chaque individu un revenu monétaire périodique de manière inconditionnelle, ces propositions peuvent viser des objectifs aussi divers que potentiellement contradictoires (ex. : rationaliser la protection sociale, flexibiliser le marché du travail, lutter contre le productivisme, favoriser le travail hors emploi, etc.), qui se traduisent eux-mêmes par des modalités d'application tout aussi variées (en termes de montants proposés, de sources de financement, du maintien ou non des prestations sociales existantes ou encore de la possibilité de cumuler ce revenu avec d'autres revenus, par exemple), ainsi que par des appellations différentes (ex. : « revenu de base », "revenu de citoyenneté», "dividende social», "allocation universelle», etc.). Et pourtant, pour bon nombre de partisans du revenu inconditionnel, ces différences sont secondaires par rapport au principe même du revenu inconditionnel, dont la défense constitue leur priorité, même si cela implique des alliances tactiques temporaires avec des concurrents ou «ennemis » politiques5. Et symétriquement, de nombreux opposants rejettent en bloc toute forme de revenu inconditionnel en arguant par exemple qu'elles sont toutes «fondamentalement libérales» (Horny, 2016).

Nous souhaiterions toutefois approfondir ici l'hypothèse qu'il n'existe pas « un » revenu inconditionnel, mais des projets politiques très différents qui se fondent sur des considérations sociopolitiques incompatibles, particulièrement dans leur rapport au système économique capitaliste.

4. À ce sujet, voir notamment la « liste des partisans du revenu de base » recensée par Wikipedia : https://fr.wikipedia.org/wiki/Liste_de_partisans_du_revenu_de_base (consultéle 26/01/2017).

5. Le Mouvement français pour un revenu de base (MFRB) se définit ainsi comme un mouvement «transpartisan»: http://revenudebase.info/qui-sommes-nous/ (consulté le 26/01/2017). 


\section{Le revenu inconditionnel par ses discours}

Pour ce faire, nous nous sommes livré à une analyse lexicométrique de neuf textes défendant chacun une version particulière de revenu inconditionnel. Comme nous l'avons déjà mentionné, six d'entre eux sont tirés d'auditions réalisées pour le compte d'une mission d'information du Sénat français réalisée en 2016 (Percheron, 2016). Au total, ce sont près de soixante intervenants qui sont venus y exprimer leur position (ou, plus généralement, celle de leur organisation) par rapport au revenu inconditionnel. Parmi ceux-ci, nous n'avons toutefois gardé que ceux qui en défendaient concrètement une forme spécifique, à l'exclusion donc de ceux qui avaient été simplement invités à venir donner leur avis (ou celui de leur organisation) sur son principe et/ou telle application concrète. Ce premier sous-corpus est ainsi constitué de six textes produits par les auteurs suivants :

- Marc de Basquiat est ingénieur et docteur en économie. M. de Basquiat a une carrière de cadre dirigeant dans le privé en marge de laquelle il a développé un intérêt pour le revenu inconditionnel. Cofondateur du Mouvement français pour un revenu de base (MFRB), il est également membre de l'Association pour l'instauration d'un revenu d'existence (AIRE), qu'il préside depuis 2014, et du BIEN. En 2014, il a publié le rapport Liber, un revenu de liberté pour tous, avec le philosophe libéral Gaspard Kœnig (voir ci-dessous);

- Jean-Éric Hyafil est doctorant en économie. Cofondateur du MFRB et coordinateur de l'ouvrage Revenu de base, un outil pour construire le Xxıe siècle, J.-É. Hyafil s'est d'abord intéressé au revenu de base dans une perspective de "décroissance », avant de l'envisager aujourd'hui surtout comme une solution à la « révolution numérique» en cours et à ses impacts sur le travail;

- Gaspard Kœnig est philosophe. Outre l'enseignement de la philosophie, G. Kœnig a également travaillé pour Christine Lagarde au ministère de l'Économie, puis à la Banque européenne de restructuration et de développement (BERD) à partir de 2009. En 2013, il a fondé le think tank libéral GenerationLibre, qui a notamment publié le rapport coécrit avec M. de Basquiat, Liber, un revenu de liberté pour tous (voir ci-dessus);

- Emmanuel Amon et Sébastien Rouchon sont représentants du Centre des jeunes dirigeants d'entreprise (CJD). Le CJD se targue d'être le plus ancien mouvement patronal de France. Créé en 1938, il compte aujourd'hui environ 4500 adhérents. Outre des missions de soutien à ses membres dirigeants d'entreprise, le CJD se veut aussi le lieu de réflexion et d'expérimentation d'un « libéralisme responsable ». En 2011, cela l'a notamment amené à inclure une proposition de revenu inconditionnel dans un projet de refonte globale du système social et fiscal français, intitulé Objectif Oïkos; 
- Baptiste Mylondo est professeur d'économie et de philosophie politique. Partisan de la décroissance, B. Mylondo a écrit et dirigé plusieurs ouvrages sur le sujet. Il a également cofondé et cogéré une coopérative de distribution agricole alternative entre 2004 et 2008 . Depuis lors, il se consacre toutefois surtout au revenu inconditionnel, notamment, depuis 2011, dans le cadre du collectif Pour un revenu social (POURS);

- Philippe Van Parijs est philosophe et économiste. Professeur à l'université catholique de Louvain (UCL), en Belgique francophone, P. Van Parijs est l'un des précurseurs du regain d'intérêt pour l'idée de revenu inconditionnel dans les cercles «post-marxistes» du courant des années 1980. Il développe d'abord ses positions au sein du collectif Charles Fourier créé en 1984 à Louvain-la-Neuve, puis du BIEN créé deux ans plus tard et dont il reste l'un des principaux penseurs et animateurs.

Ce premier sous-corpus est déjà intéressant dans la mesure où il regroupe des partisans du revenu inconditionnel généralement classés plutôt à gauche (J.-É. Hyafil, B. Mylondo et P. Van Parijs) et plutôt à droite (M. de Basquiat, G. Kœnig et le CJD). Il a en outre l'énorme avantage de répondre aux mêmes conditions de production, ce qui en facilite évidemment la comparaison. Il est néanmoins relativement pauvre du point de vue de la diversité des propositions existantes en matière de revenu inconditionnel, notamment parce qu'il n'inclut aucun représentant des principaux courants "radicaux», à l'exclusion, peutêtre, de B. Mylondo (ce qui en soi est déjà un indice intéressant du cercle restreint dans lequel le débat légitime sur le revenu inconditionnel est enfermé). C'est pourquoi nous avons décidé de le compléter par trois textes représentatifs de courants plus critiques sur l'économie capitaliste. Les deux premiers sont tirés d'un numéro spécial de la revue Mouvements (Cottin-Marx, Garda, Mylondo éd., 2013) consacré aux propositions de revenu inconditionnel. On y retrouve un texte de Bernard Friot (pour le courant «communiste») et un autre de Paul Ariès (pour le courant «décroissantiste») :

- B. Friot est sociologue et économiste du travail. Marxiste revendiqué, il s'appuie sur une lecture particulière de la sécurité sociale et de la fonction publique comme institutions anticapitalistes pour en proposer l'extension à l'ensemble de l'économie sous la forme d'un «salaire à vie » dont la proposition est portée et développée par le réseau d’éducation populaire Réseau salariat ${ }^{6}$;

- P. Ariès est politologue et écrivain. Considéré comme un des penseurs de référence de la «décroissance» en France, il s'est notamment prononcé en faveur d'un revenu inconditionnel versé sous une forme partiellement démonétarisée.

Enfin, le troisième et dernier texte a été publié par la revue Multitudes et il est cosigné par Antonella Corsani (sociologue et économiste du travail) et Maurizio Lazzarato (sociologue et philosophe). Ces deux auteurs sont des tenants

6. Voir le site http://www.reseau-salariat.info/?lang=fr (consulté le 26/01/2017). 
de longue date de la thèse du «capitalisme cognitif» et de la nécessité que son émergence impose de repenser la question du revenu et du travail7.

Tous ces textes ont ainsi pour caractéristique commune d'incarner un argumentaire en faveur d'une version particulière de revenu inconditionnel. Ils sont également de tailles comparables (sauf celui de B. Mylondo, qui est particulièrement court, voir tableau 1), ce qui permet de comparer ce qui est mis en avant (et comment) par ces différents auteurs dans une contrainte de place similaire.

Tableau 1. Statistiques du corpus « revenu inconditionnel»

\begin{tabular}{l|c|c}
\hline \multicolumn{1}{c|}{ Textes } & Occurrences $^{\mathrm{a}}$ & Nombre de formes lexicales $^{\mathrm{b}}$ \\
\hline Ariès & 2314 & 717 \\
\hline de Basquiat & 2504 & 841 \\
\hline CJD & 3401 & 922 \\
\hline Corsani/Lazzarato & 3841 & 937 \\
\hline Friot & 5119 & 1054 \\
\hline Hyafil & 2435 & 736 \\
\hline Kœnig & 5631 & 1452 \\
\hline Mylondo & 634 & 248 \\
\hline Van Parijs & 3306 & 1009 \\
\hline Tous & 29185 & 7916 \\
\hline
\end{tabular}

a. Nombre total d'occurrences (mots) dans le texte.

b. Nombre de formes lexicales différentes dans le texte.

Pour l'analyse, nous avons eu recours au logiciel de lexicométrie TXM8 ${ }^{8}$ La lexicométrie permet un décentrement par rapport aux textes analysés, ainsi qu'une objectivation relative de l'analyse, qui nous sont particulièrement utiles au moment de traiter de la signification politique de corpus de textes en concurrence politique (Bonnafous, Tournier, 1995; Deroubaix, Gobin, 2009). Elle permet également de prêter une grande attention aux termes mobilisés, qui sont importants en eux-mêmes par les choix et les imaginaires9 9 politiques qui les fondent et qu'ils fondent en retour. Mais ils sont aussi (et peut-être encore plus) importants

7. Par « capitalisme cognitif », il faut entendre une nouvelle phase de l'histoire du capitalisme où la connaissance devient le facteur de production prédominant, notamment au détriment du «travail ». Pour plus de détails sur cette thèse, lire, entre autres, l'ouvrage de Yann Moulier-Boutang (Moulier-Boutang, 2007).

8. Voir le site http://textometrie.ens-lyon.fr/ (consulté le 26/01/2017) et l'article de Serge Heiden, Jean-Philippe Magué et Bénédicte Pincemin (Heiden et al., 2010).

9. Nous préférons le terme «imaginaire» au terme «idéologie» parce qu'il est moins connoté et surtout plus englobant. Plusieurs idéologies peuvent ainsi s'inscrire dans un même imaginaire, à l'image des idéologies socialiste et libérale par exemple, qui participent toutes les deux d'un imaginaire plus large de croyance en la possibilité et la désirabilité d'une maîtrise rationnelle de la nature (Castoriadis, 1975 ; Charaudeau, 2005). 
dans les réseaux lexicaux qu'ils dessinent et qui contribuent également à en déterminer le sens et les effets (Boutet, 2016; Deroubaix, Gobin, 2009). Enfin, parce que les mots constituent «les briques du discours» (Deroubaix, Gobin, 2009) à partir desquels il est possible de retracer certaines logiques discursives plus larges, tout en sachant bien évidemment que l'analyse des mots mis en évidence par les calculs statistiques doit se coupler avec une connaissance préalable de ces textes et plus largement des travaux de leurs auteurs.

En outre, étant donné que cet objet est encore peu balisé politiquement et que nous sommes toujours dans une phase d'explications et de définitions en concurrence, les outils contrastifs insérés dans TXM sont particulièrement adaptés ${ }^{10}$. Enfin, les recherches sur ce thème à travers l'abord des jeux et tensions discursifs auxquels il donne lieu sont encore quasi inexistantes ${ }^{11}$. Précisons toutefois qu'il est relativement inhabituel de mobiliser la lexicométrie pour l'analyse d'un corpus de si faible ampleur : beaucoup de ses outils statistiques ne conviennent pas pour un volume de données si modeste. Dès lors, l'intérêt de cet article est aussi d'illustrer l'apport possible de la lexicométrie pour l'analyse de « petits» corpus.

\section{Résultats}

\section{Des dénominations plurielles et contestées}

La simple analyse de la façon dont les projets de revenu inconditionnel sont désignés dans notre corpus indique déjà l'existence d'une pluralité de représentations potentiellement antagoniques dans ce domaine. En effet, si l'on se réfère à la dénomination privilégiée par chaque auteur (voir tableau 3), on

10. Les principaux outils lexicométriques mobilisés dans le cadre de cette étude sont : l'analyse factorielle des correspondances (AFC), qui permet une visualisation graphique (sur un plan à deux axes) des relations de similarités/dissimilarités statistiques entre les vocabulaires de plusieurs textes. Concrètement, plus deux textes sont proches dans l'espace (ou au moins présents dans le même cadran), plus leurs profils lexicaux sont statistiquement similaires. À l'inverse, plus ils sont éloignés (ou dans des cadrans différents), plus cela signifie qu'ils mobilisent des vocabulaires différents; le calcul des fréquences, c'est-à-dire du nombre total (ou relatif) d'apparitions d'une forme lexicale donnée dans l'ensemble du corpus ou dans une partie de celui-ci ; le calcul des spécificités, c'est-à-dire le degré de sur-emploi, de sous-emploi ou d'emploi banal d'une (ou plusieurs) forme(s) lexicale(s) donnée(s) au sein d'une sous-section du corpus, par rapport à l'ensemble de celui-ci ; la mise en liste organisée des concordances, c'est-à-dire le listage de l'ensemble des cotextes immédiats (à gauche et à droite) d'une forme lexicale (ou segment répété) donnée, et ce afin d'en saisir et d'en quantifier les différents usages, que ce soit pour l'ensemble du corpus ou au sein de sous-sections préalablement définies. Pour plus de détails sur ces outils et sur les outils lexicométriques en général, se référer à l’ouvrage de Ludovic Lebart et André Salem (Lebart, Salem, 1994).

11. Une exception notable peut être trouvée chez Jérémy Dodeigne, Min Reuchamps, Audrey Vandeleene et al. (Dodeigne, Reuchamps, Vandeleene et al., 2017), qui ont consacré un article à l'impact de l'usage des métaphores dans l'évaluation des propositions d'allocation universelle. L'article se contente néanmoins de constater qu'il existe un impact (positif) des métaphores dans l'évaluation qui est faite des propositions d'allocation universelle, mais sans l'expliquer. 
voit que le conflit politique se joue sur trois dénominations concurrentes: "revenu», "salaire» et "allocation». Dans un premier temps, avant d'avancer plus loin dans l'analyse, en s'appuyant sur la connaissance des pratiques discursives usuelles dans notre imaginaire politique contemporain, on peut suggérer que le terme de "revenu » permet de détacher le travail de sa rémunération ou la rémunération du travail (on peut en effet tirer un revenu de multiples autres activités que du «travail»). Au contraire, le terme «salaire» est indissociable des notions de travail (et travailleur), rémunération et production de la richesse. Quant au terme "allocation », il suggère une rémunération marginale qui prend sa place parmi d'autres types de rémunérations.

Ensuite, on constate que ce corpus ne mobilise pas moins de quinze appellations différentes (voir tableau 2) chargées de connotations qui sont à chaque fois lourdes de sens sur le plan politique et philosophique (référence à la citoyenneté, à l'universalisme, à l'existence, etc.). Or, chaque texte (ou presque) mobilise une appellation différente en priorité (voir tableau 3), seuls « revenu garanti » et « revenu de base » étant utilisés en priorité par deux textes différents.

Tableau 2. Dénominations concurrentes du revenu inconditionnel (classées par fréquences d'apparition décroissantes dans des textes différents)

\begin{tabular}{l|c|c|l}
\hline \multicolumn{1}{c|}{ Appellations } & FTOT & Textes (nombre) & \multicolumn{1}{c}{ Textes (auteurs) } \\
\hline revenu de base & 87 & 6 & $\begin{array}{l}\text { Basquiat, CJD, Friot, Hyafil, } \\
\text { Kœnig, Van Parijs }\end{array}$ \\
\hline revenu d'existence & 19 & 5 & $\begin{array}{l}\text { Ariès, Corsani/Lazzarato, } \\
\text { Basquiat, CJD, Kœnig }\end{array}$ \\
\hline revenu inconditionnel & 7 & 4 & $\begin{array}{l}\text { Ariès, Kœnig, Mylondo, } \\
\text { Van Parijs }\end{array}$ \\
\hline allocation universelle & 21 & 3 & Basquiat, Kœnig, Van Parijs \\
\hline revenu social & 7 & 3 & Ariès, Corsani, Van Parijs \\
\hline revenu universel & 26 & 2 & CJD, Kœnig \\
\hline revenu garanti & 19 & 2 & Ariès, Corsani \\
\hline salaire socialisé & 5 & 2 & Ariès, Friot \\
\hline dividende social & 2 & 2 & Ariès, Van Parijs \\
\hline salaire à vie & 10 & 1 & Friot \\
\hline $\begin{array}{l}\text { dotation inconditionnelle } \\
\text { d'autonomie }\end{array}$ & 2 & 1 & Ariès \\
\hline revenu de citoyenneté & 1 & 1 & Ariès \\
\hline revenu citoyen & 1 & 1 & Basquiat \\
\hline revenu de participation & 1 & 1 & Mylondo \\
\hline salaire universel & 1 & 1 & Friot \\
\hline dividende territorial & 1 & 1 & Van Parijs \\
\hline & & &
\end{tabular}


Certes, on remarquera que c'est de loin le substantif « revenu» qui est le plus courant et qui connaît le plus de déclinaisons. En outre, un nombre réduit d'appellations circule entre plusieurs textes, cinq d'entre elles se retrouvant notamment dans plus de trois textes (voir tableau 2). Néanmoins, cette circulation se fait surtout entre les textes tirés des auditions sénatoriales sur le «revenu de base». Et surtout, elle recouvre deux logiques distinctes, à savoir : - une logique consensuelle, qui caractérise les textes issus des auditions du Sénat français, où différentes appellations peuvent être mobilisées soit comme de purs substituts (1), soit comme des variantes d'une même idée (2) :

1. Le centre des jeunes dirigeants travaille sur le revenu d'existence, encore appelé revenu de base ou revenu universel. (Amon, Rouchon, 2016, p. 343)

2. C'est ce qu'il [Joseph Charlier] appelait le dividende territorial [...] trois débats publics se sont tenus sur ce revenu de base. (Van Parijs, 2016, p. 220)

- une logique contradictoire, présente uniquement dans les textes de P. Ariès et de B. Friot, où différentes appellations sont mobilisées, mais cette fois pour signifier explicitement l'existence de projets politiques différents, voire antagonistes :

3. Nous ne devons pas davantage être dupes lorsque nos adversaires de droite comme Alain Madelin, Christine Boutin ou Dominique de Villepin parlent de «dividende social». (Ariès, 2013, p. 24)

4. Pourquoi régresser par l'instauration d'un revenu de base qui, laissant intacts le marché du travail, la valeur travail et la propriété lucrative, s'inscrit comme correctif dans la domination capitaliste? (Friot, 2013, p. 68)

On peut également relever le cas particulier du texte de A. Corsani et M. Lazzarato qui, sans polémiquer directement avec d'autres appellations, utilise surtout celle de «revenu garanti », relativement peu utilisée par d'autres auteurs, mais en la définissant de façon si restrictive qu'elle exclut de facto beaucoup d'autres formules :

5. Le revenu garanti ne doit surtout pas s'inscrire dans une logique de redistribution mais dans une logique subversive de dépassement radical d'une répartition de la richesse fondée sur le capital et le travail. (Corsani, Lazzarato, 2002, p.184)

\section{Trois imaginaires en tension}

Ces premiers résultats semblent donc confirmer l'existence de représentations conflictuelles du revenu inconditionnel. Or, l'analyse factorielle des correspondances (AFC, réalisée sur les formes avec un seuil de fréquence minimum de 9) permet d'aller encore plus loin dans ce constat, en comparant cette fois les profils lexicaux respectifs des différents textes soumis à l'analyse. C'est ainsi 
Tableau 3. Répartition par texte des dénominations concurrentes de revenu inconditionnel

\begin{tabular}{l|c|l|c|l|c}
\hline \multicolumn{1}{c|}{ Ariès } & FQ & \multicolumn{1}{c|}{ Basquiat } & FQ & \multicolumn{1}{c|}{ CJD } & FQ \\
\hline revenu garanti & 11 & revenu d'existence & 15 & revenu universel & 15 \\
\hline revenu social & 5 & revenu de base & 3 & revenu de base & 9 \\
\hline $\begin{array}{l}\text { dotation inconditionnelle } \\
\text { d'autonomie }\end{array}$ & 2 & $\begin{array}{l}\text { allocation } \\
\text { universelle }\end{array}$ & 3 & revenu d'existence & 1 \\
\hline revenu d'existence & 1 & & & & \\
\hline revenu citoyen & 1 & & & & \\
\hline salaire socialisé & 1 & & & & \\
\hline dividende social & 1 & & & \\
\hline
\end{tabular}

\begin{tabular}{l|c|l|c|l|c}
\hline \multicolumn{1}{c|}{ Corsani/Lazzarato } & FQ & \multicolumn{1}{c|}{ Friot } & FQ & \multicolumn{1}{c}{ Hyafil } & FQ \\
\hline revenu garanti & 8 & salaire à vie & 10 & revenu de base & 39 \\
\hline revenu d'existence & 1 & salaire socialisé & 4 & revenu universel & 1 \\
\hline revenu social & 1 & salaire universel & 1 & & \\
\hline & & revenu de base & 1 & & \\
\hline
\end{tabular}

\begin{tabular}{l|c|l|c|l|c}
\hline \multicolumn{1}{c|}{ Kœnig } & FQ & \multicolumn{1}{c|}{ Mylondo } & FQ & \multicolumn{1}{c|}{ Van Parijs } & FQ \\
\hline revenu de base & 28 & $\begin{array}{l}\text { revenu } \\
\text { inconditionnel }\end{array}$ & 3 & $\begin{array}{l}\text { allocation } \\
\text { universelle }\end{array}$ & 17 \\
\hline revenu universel & 8 & $\begin{array}{l}\text { revenu de } \\
\text { participation }\end{array}$ & 1 & revenu de base & 3 \\
\hline revenu de base universel & 4 & & & $\begin{array}{l}\text { revenu } \\
\text { inconditionnel }\end{array}$ & 2 \\
\hline revenu universel de base & 2 & & & dividende social & 1 \\
\hline revenu d'existence & 1 & & & $\begin{array}{l}\text { dividende } \\
\text { territorial }\end{array}$ & 1 \\
\hline revenu inconditionnel & 1 & & & & 1 \\
\hline allocation universelle & 1 & & & & \\
\hline
\end{tabular}

que l'axe horizontal, sur la figure 1, oppose l'ensemble des textes extraits des auditions sénatoriales françaises (à droite) aux trois textes représentatifs des courants plus radicaux en matière de revenu inconditionnel (à gauche), ce qui confirme l'opposition déjà identifiée lors de l'analyse des dénominations. L'analyse des contributions ${ }^{12}$ de chaque texte à ce premier axe montre toutefois que c'est d'abord et avant tout le texte de B. Friot qui explique cette première opposition (à plus de $50 \%$ ) selon une logique de rupture économique (chez B. Friot) avec le système capitaliste versus une logique de réforme/adaptation à

12. Par le calcul des «contributions absolues» de chacun des textes à l'axe examiné. 
celui-ci (dans les autres textes issus du rapport sénatorial13). L'analyse du vocabulaire le plus spécifiquement lié à chacun de ces deux pôles (voir tableau 4) montre en effet :

- pour les textes de M. de Basquiat, CJD, J.-É. Hyafil, G. Kœnig et P. Van Parijs, la primauté des termes « revenu » et «base», mais aussi et surtout un registre essentiellement technique et pragmatique qui renvoie à du problem-solving ${ }^{14}$ en matière de politique fiscale et de protection sociale (ex. : "système», «euro», «RSA», «impôts», « revenus »);

- pour le texte de $B$. Friot, un registre essentiellement salarial qui renvoie à des dispositifs institutionnels visant à encadrer et (ré)instituer la relation de travail dans une société où le salaire absorbe complètement le revenu du capital (ex. : «qualification », « salaire», «cotisations», « convention», «poste »).

Si l'on examine ensuite le second contraste (axe vertical) révélé par la deuxième dimension de l'AFC (qui doit être lu comme une « correction » du premier contraste), on constate qu'il concerne essentiellement les textes de B. Friot d'un côté et de P. Ariès et A. Corsani/M. Lazzarato de l'autre (à plus de $90 \%$ !), selon une logique de rupture économique (chez B. Friot) versus une logique de rupture avec l'économie elle-même (chez P. Ariès et A. Corsani/M. Lazzarato). En effet, chez ces derniers, les spécificités (voir tableau 4) relèvent d'un registre essentiellement existentiel qui renvoie plutôt à des questions de valeur d'usage (ex. : «gratuité», «communs», «dons», «formes», «vie»).

On serait donc en présence non pas de deux, mais bien de trois pôles discursifs (et plus largement imaginaires) distincts, avec :

- un pôle capitaliste (à droite de l'axe vertical);

- un pôle anti-capitaliste (B. Friot);

- un pôle anti-économique (P. Ariès et A. Corsani/M. Lazzarato).

13. À l'exception toutefois du texte de B. Mylondo qui s'inscrit quant à lui dans un registre essentiellement moral (ex. : «pauvreté», « reconnaître», «injuste»). L'analyse de ses contributions à l'AFC montre néanmoins qu'il ne participe à la construction d'aucun des deux axes, probablement en raison de sa trop faible longueur par rapport aux autres textes. C'est pourquoi nous ne l'intégrons pas dans la suite de l'analyse.

14. Littéralement « résolution de problème ». II s'agit d'un terme anglais qui désigne une démarche pragmatique et technique où la quête du résultat prime sur la recherche des causes ou plus largement sur toute forme de considération éthique et/ou politique. Le politologue canadien Robert W. Cox l'oppose par exemple à une démarche de problem-posing qui est selon lui au fondement de toute théorie critique (Cox, 1981). 
Figure 1. AFC réalisée sur une partition par auteurs du corpus « revenu inconditionnel»

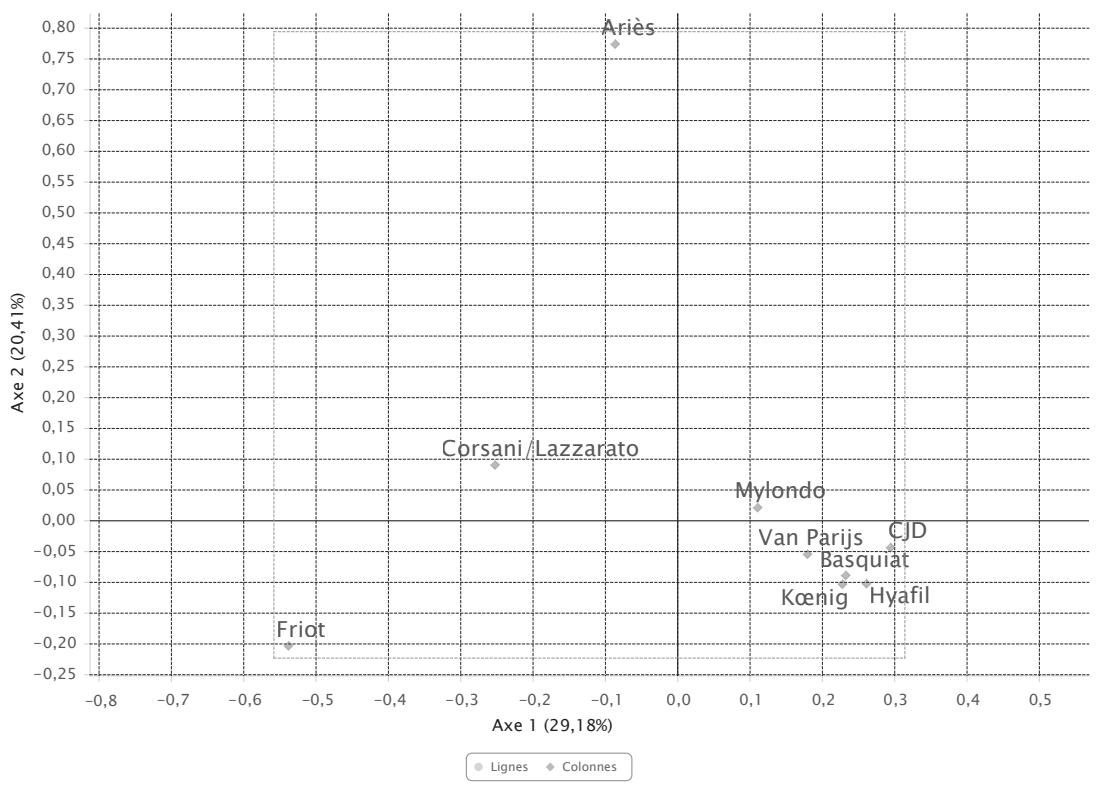

Tableau 4. Listes des 10 mots pleins les plus spécifiques aux trois pôles identifiés par l'AFC

\begin{tabular}{l|c|c}
\hline Pôle 1 (Friot) & Fréquence & Spécificité \\
\hline valeur & 71 & 49,1 \\
\hline travail & 222 & 25,8 \\
\hline qualification & 34 & 25,7 \\
\hline salaire & 43 & 21,7 \\
\hline cotisation & 29 & 19,8 \\
\hline convention & 24 & 18,2 \\
\hline capitaliste & 29 & 14,9 \\
\hline économique & 34 & 10,8 \\
\hline abstrait & 13 & 9,8 \\
\hline poste & 12 & 9,1 \\
\hline
\end{tabular}

\begin{tabular}{l|c|c}
\hline $\begin{array}{l}\text { Pôle 2 (de Basquiat, } \\
\text { CJD, Hyafil, Kœnig, } \\
\text { Van Parijs) }\end{array}$ & Fréquence & Spécificité \\
\hline base & 97 & 18,8 \\
\hline revenu & 234 & 8,9 \\
\hline système & 56 & 8,8 \\
\hline euros & 52 & 8 \\
\hline allocation & 31 & 7,1 \\
\hline RSA & 52 & 6,2 \\
\hline idée & 25 & 5,7 \\
\hline impôt & 65 & 5 \\
\hline revenus & 35 & 4,6 \\
\hline gens & 19 & 4,3 \\
\hline
\end{tabular}




\begin{tabular}{l|c|c}
$\begin{array}{l}\text { Pôle 3 } \\
\text { (Ariès, Corsani/Lazzarato) }\end{array}$ & Fréquence & Spécificité \\
\hline garanti & 20 & 11,6 \\
\hline combat & 11 & 7,4 \\
\hline richesse & 12 & 6,5 \\
\hline gratuité & 20 & 6,4 \\
\hline communs & 14 & 6,3 \\
\hline don & 9 & 6,1 \\
\hline formes & 19 & 5,9 \\
\hline salarié & 19 & 5,9 \\
\hline vie & 72 & 5,6 \\
\hline production & 32 & 5,6 \\
\hline
\end{tabular}

\section{Le revenu inconditionnel comme enjeu distributif ou productif?}

L'analyse des «index hiérarchiques " $^{15}$ de chaque texte pris individuellement (voir tableau 5) permet toutefois d'enrichir encore un peu plus ces observations, en ajoutant cette fois une troisième ligne de fracture au sein du corpus entre ceux (la majorité) pour qui la question du revenu inconditionnel semble d'abord une question de (re) distribution du revenu et/ou des richesses disponibles (P. Ariès, M. de Basquiat, J.-É. Hyafil, G. Kœnig, B. Mylondo et P. Van Parijs) et les autres (minoritaires) pour qui il s'agirait d'abord d'une question de rapport au travail (A. Corsani/M. Lazzarato, B. Friot et CJD). Dans un cas comme dans l'autre, on retrouve toutefois les distinctions révélées ci-dessus, l'analyse des concordances de «travail» dans le second groupe, par exemple, permettant de voir comment chacun de ces textes envisage de redéfinir le «travail » par le revenu inconditionnel depuis un des trois pôles (capitaliste, anti-capitaliste, anti-économique) identifiés grâce à l'AFC.

Deux conceptions du «travail » pour trois perspectives antagoniques du revenu inconditionnel

C'est ainsi que pour B. Friot, par exemple, le «travail » est d'abord une convention sociale :

On travaille lorsque l'activité productrice de valeur d'usage [...] se double d'une production de valeur économique. [...] L'activité devient donc du travail lorsqu'elle s'inscrit dans des institutions qui, par convention, ajoutent à la valeur d'usage une valeur économique. (Friot, 2013, p.60)

15. Un «index hiérarchique » est un tableau de fréquences, trié par fréquence décroissante. 
Tableau 5. Liste des 10 mots pleins les plus fréquents dans chaque texte analysé

\begin{tabular}{l|l|l|l|l}
\hline Ariès & Basquiat & CJD & $\begin{array}{l}\text { Corsani/ } \\
\text { Lazzarato }\end{array}$ & Friot \\
\hline revenu & revenu & revenu & travail & travail \\
\hline gratuité & existence & travail & vie & valeur \\
\hline combat & impôt & faut & temps & salaire \\
\hline garanti & euros & emploi & production & qualification \\
\hline social & système & universel & revenu & cotisation \\
\hline biens & revenus & entreprise & salarié & capitaliste \\
\hline communs & être & collaborateurs & entreprise & convention \\
\hline don & RSA & créer & formes & économique \\
\hline être & niveau & base & capital & temps \\
\hline services & prestations & emplois & emploi & marché \\
\hline
\end{tabular}

\begin{tabular}{l|l|l|l}
\hline Hyafil & Kœnig & Mylondo & Van Parijs \\
\hline revenu & revenu & revenu & allocation \\
\hline base & base & tous & universelle \\
\hline impôt & système & pauvreté & idée \\
\hline RSA & euros & emploi & emplois \\
\hline euros & impôt & RSA & débat \\
\hline revenus & RSA & vie & sociale \\
\hline activité & gens & être & modèle \\
\hline autonomie & manière & logique & dire \\
\hline montant & universel & reconnaître & revenu \\
\hline négatif & travail & travail & manière \\
\hline
\end{tabular}

À l'image de l'ensemble des résultats présentés dans cet article, les résultats de ce tableau ont été obtenus sans recours à la lemmatisation. Il s'agit davantage d'une position de principe liée à la crainte de voir se perdre des informations ou subtilités importantes que d'un choix inspiré par l'objet et/ou l'objectif de la recherche. 
Or, selon lui, il est possible de distinguer aujourd'hui non pas une, mais deux conventions antagonistes du travail :

L'une qui veut que «travailler», ce soit «être sur le marché du travail» : c'est la convention capitaliste, dominante. L'autre, anticapitaliste, qui veut que «travailler» ce soit «avoir une qualification personnelle et donc un salaire à vie» : elle est dominée mais bien réelle du fait de sa présence dans la fonction publique et de son extension récente aux retraités. (Friot, 2013, p. 64-65)

Dès lors, pour B. Friot, la question n'est pas de savoir si on est pour ou contre «le» travail, mais bien pour ou contre telle convention particulière du travail. C'est pourquoi, chez lui, le terme «travail» est toujours mobilisé en référence à une convention précise et/ou à ses enjeux sous-jacents.

À l'inverse, pour A. Corsani et M. Lazzarato, le «travail » n'est envisagé que dans sa signification étroitement capitaliste, et encore, principalement en référence à une phase précise de l'histoire du capitalisme, le «fordisme». On le voit à travers la multiplication des références au «travail industriel » ou encore au «travail salarié» (entendu comme travail salarié en entreprise), mais aussi à travers de nombreux usages où «travail » est utilisé comme synonyme de «emploi » (un abus de langage qui s'explique par le fait que dans le fordisme, l'emploi constituait effectivement la matrice principale du travail). Or, selon les auteurs, il est d'autant plus urgent de se libérer de ce «travail» qu'il n'est plus au centre du processus d'accumulation, lequel porte de plus en plus sur la «vie » elle-même :

[...] nous sommes confrontés à une accumulation capitaliste qui ne se fonde plus seulement sur l'exploitation du travail dans le sens industriel du terme, mais sur celle de la connaissance, du vivant, de la santé, du temps libre, de la culture, des ressources relationnelles entre individus (communication, socialisation, sexe), de l'imaginaire, de la formation, de l'habitat, etc. (Corsani, Lazzarato, 2002, p. 178)

Cela implique donc que le «travail » (étroitement conçu) ne puisse plus offrir une base solide de droits et de revenu, ni davantage un point de départ nécessaire et encore moins suffisant pour penser des luttes en faveur de l'émancipation :

Nous devons résister aux politiques néolibérales, mais à partir de l'affirmation, en positif, de la puissance de cette «vie». Autrement dit, nous devons penser audelà du travail et de ses formes classiques d'exploitation [...]. (Corsani, Lazzarato, 2002, p. 179)

C'est donc d'abord et avant tout le déplacement du processus d'accumulation capitaliste sur le terrain de la «vie» elle-même qui semble justifier ici le dépassement de ce que les auteurs nomment la "société du travail». Par contre, si l'on prend le texte de P. Ariès, qui partageait également la même insistance sur les dimensions existentielles de la lutte pour un revenu inconditionnel (voir tableau 3), celles-ci semblent plutôt trouver leur justification en elles-mêmes et non pas tant dans le caractère obsolète du «fordisme» et 
des relations de travail qui l'accompagnaient. Le texte ne mentionne d'ailleurs presque pas le «travail» (3 occurrences seulement) et lorsque c'est le cas, c'est surtout pour en contester la désirabilité intrinsèque : il s'agit d'« en finir avec la centralité du travail dans nos existences».

Enfin, dans le texte du CJD, on retrouve une volonté similaire à celle de B. Friot de distinguer «travail» et «emploi », mais cette fois dans l'optique de lever les obstacles à une marchandisation complète du travail qui restent associés au cadre légal de l'emploi. C'est ainsi qu'en parallèle de la distinction opérée entre ces deux notions,

[...] il existe une différence fondamentale entre emploi et travail. L'emploi, c'est la promesse du chef d'entreprise; c'est quelque chose que l'entrepreneur - ou l'auto-entrepreneur - a la capacité de créer. Le travail est une notion beaucoup plus large : on peut travailler sans avoir de véritable emploi, tout comme on peut travailler en ayant de multiples emplois. (Amon, Rouchon, 2016, p. 345)

le texte insiste surtout sur la nécessité d’encourager le «travail » :

Le travail est l'un des fondements de notre vie. Il est absolument nécessaire de redonner le goût du travail à tout le monde. (Amon, Rouchon, 2016, p.347)

Mais il s'agit ici à nouveau d'un « travail » étroitement conçu comme activité, voire comme objet strictement marchand dont le développement est notamment entravé par l'emploi et les coûts qui lui sont associés :

Moi, j’ai du travail à fournir, mais je ne sais pas comment le rémunérer : certains de mes collaborateurs sont surchargés, mais comme je ne fais quasiment pas de bénéfice, je ne peux transformer ces gisements de travail en emplois. (Amon, Rouchon, 2016, p. 348)

Aujourd'hui, il y a beaucoup de travail qu'on ne peut payer 15 euros de l'heure. Quand on compare le SMIC, les charges et le temps administratif associés à un emploi, d'une part, au coût de la robotisation, d'autre part, le travail n'est pas compétitif. (Amon, Rouchon, 2016, p. 350)

Dans ce contexte, le revenu inconditionnel a donc le double avantage 1) de fournir un filet de sécurité minimal en remplacement des protections associées à l'emploi, considérées comme obsolètes et 2) de libérer le développement du travail, mais dans un sens qui reste attaché à une activité étroitement marchande :

Le revenu universel va nous permettre non seulement de couvrir ce premier besoin [de survie], mais aussi de donner le goût, l'envie, à tout un chacun de développer du travail. (Amon, Rouchon, 2016, p.346-347)

À l'heure où le débat sur la mise en place d'une forme de revenu inconditionnel prend de l'ampleur en Europe, nous avons voulu interroger la façon dont certaines des propositions les plus débattues s’inscrivaient dans des 
projets et des imaginaires politiques différents, voire antagonistes. L'engouement qui entoure cette idée nous semble en effet largement dû à une confusion problématique entre des modèles et des objectifs extrêmement variés. À travers une analyse lexicométrique de discours fondant et légitimant des propositions concrètes de revenu inconditionnel, nous avons donc voulu non seulement mettre à jour cette pluralité discursive, mais aussi montrer comment elle se construisait dans et à travers le langage.

Le premier constat est celui d'une distinction claire entre les propositions qui s'inscrivent dans le cadre économique actuel et celles qui visent à le remettre en question. Cette distinction est apparue à la fois d'un point de vue statistique, notamment grâce à l'AFC (voir figure 1), mais aussi et surtout d'un point de vue qualitatif avec dans le premier cas des vocabulaires essentiellement pragmatiques et techniques (dans les textes issus des auditions sénatoriales sur le «revenu de base»), et dans le second des vocabulaires de nature plus abstraite et conceptuelle (chez P. Ariès, A. Corsani/M. Lazzarato et B. Friot).

Cette première distinction se double toutefois d'une autre distinction, apparue cette fois au sein même des propositions de rupture avec le cadre économique dominant, entre celles qui pensent cette rupture contre ce cadre capitaliste et celles qui la pensent plutôt contre l'économie elle-même. D'un côté, on trouve en effet des textes (ceux de P. Ariès et A. Corsani/M. Lazzarato) où prime un vocabulaire lié à l'existence et auxvaleurs d'usage, dépassant tout économisme, tandis que de l'autre, on trouve le texte de B. Friot où prime plutôt un vocabulaire lié à des institutions économiques (alternatives).

Enfin, en parallèle, on a pu observer également une dernière distinction propre à l'ensemble du corpus et qui oppose, quant à elle, les textes (majoritaires) dans lesquels la question du revenu inconditionnel est d'abord une question de (re)distribution de revenu, que ce soit dans une perspective radicale (chez P. Ariès) ou «réformiste» (chez M. de Basquiat, J.-É. Hyafil, G. Kœnig ou encore P. Van Parijs), aux textes (ceux de A. Corsani/M. Lazzarato, B. Friot et du CJD) pour lesquels il s'agit d'abord d'une question de rapport au travail, avec à nouveau ici trois perspectives différentes (et antagonistes) :

- le revenu inconditionnel pour se libérer du travail, nécessairement capitaliste en soi (A. Corsani/M. Lazzarato);

- le revenu inconditionnel pour libérer le travail du capitalisme (B. Friot);

- le revenu inconditionnel pour stimuler le travail tout en restant dans une perspective capitaliste (CJD).

\section{Corpus}

Amon Emmanuel, Rouchon Sébastien, 2016, «Audition de MM. Emmanuel Amon et Sébastien Rouchon, du Centre des jeunes dirigeants d'entreprise (CJD)», dans Le 
revenu de base en France : de l'utopie à l'expérimentation, rapport d'information no 35 de M. Daniel Percheron, fait au nom de la mission d'information, déposé le 13 octobre 2016, Paris, Sénat français, p. 343-350.

ARIÈs Paul, 2013, «Pour un revenu social... démonétarisé», Mouvements. Des idées et des luttes, $\mathrm{n}^{0} 73$, p. 23-27.

BASQUIAT Marc de, 2016, «Audition de M. Marc de Basquiat, président de l'Association pour l'instauration d'un revenu d'existence ", dans Le revenu de base en France: de l'utopie à l'expérimentation, rapport d’information n 35 de M. Daniel Percheron, fait au nom de la mission d'information, déposé le 13 octobre 2016, Paris, Sénat français, p. 195-200.

Collectif Charles Fourier, 1985, "L'allocation universelle », La Revue nouvelle, n 4 , p. 345-394.

CORSANI Antonella, LAZZARATO Maurizio, 2002, «Le revenu garanti comme processus constituant», Multitudes, $\mathrm{n}^{\circ} 10, \mathrm{p} .177-185$.

CotTin-Marx Simon, GARDa Julie, Mylondo Baptiste éd., 2013, Un revenu pour exister [numéro thématique], Mouvements. Des idées et des luttes, nº 73.

FRIOT Bernard, 2013, "Le salaire universel : un déjà-là considérable à généraliser », Mouvements. Des idées et des luttes, $n^{\circ} 73$, p. 60-69.

Gorz André, 2002, "Pour un revenu inconditionnel suffisant», Transversales Science Culture, nouvelle série $\mathrm{n}^{\circ} 3$.

HYAFIL Jean-Éric, 2016, «Audition de M. Jean-Éric Hyafil, du Mouvement français pour un revenu de base », dans Le revenu de base en France : de l'utopie à l'expérimentation, rapport d'information n⿳0 35 de $M$. Daniel Percheron, fait au nom de la mission d'information, déposé le 13 octobre 2016, Paris, Sénat français, p. 206-210.

K氏NIG Gaspard, 2016, «Audition de M. Gaspard Kœnig, président de GenerationLibre », dans Le revenu de base en France : de l'utopie à l'expérimentation, rapport d'information $n^{\circ} 35$ de M. Daniel Percheron, fait au nom de la mission d'information, déposé le 13 octobre 2016, Paris, Sénat français, p. 275-285.

MyLondo Baptiste, 2016, «Audition de M. Baptiste Mylondo, enseignant-chercheur à l'École de commerce et de développement 3A de Lyon, chargé de cours à SciencesPo Lyon et à Centrale Paris", dans Le revenu de base en France : de l'utopie à l'expérimentation, rapport d'information no 35 de M. Daniel Percheron, fait au nom de la mission d'information, déposé le 13 octobre 2016, Paris, Sénat français, p. 243-250.

PerCheron Daniel, 2016, Le revenu de base en France : de l'utopie à l'expérimentation, rapport d'information $n^{\circ} 35$ de $M$. Daniel Percheron, fait au nom de la mission d'information, déposé le 13 octobre 2016, Paris, Sénat français, https://www.senat.fr/ rap/r16-035/r16-0351.pdf (consulté le 26 janvier 2017).

VAN PARIJS Philippe, 2016, «Audition de M. Philippe Van Parijs, professeur à l'université catholique de Louvain, fondateur du Basic Income Earth Network», dans Le revenu de base en France : de l'utopie à l'expérimentation, rapport d'information n ${ }^{\circ} 35$ de M. Daniel Percheron, fait au nom de la mission d'information, déposé le 13 octobre 2016, Paris, Sénat français, p. 217-223.

\section{Références}

BABINET Gilles, 2016, Transformation digitale : l'avènement des plateformes, Paris, Le Passeur. 
Baudouin Thierry, Chopart Jean-Noël, Collin Michèle, Guilloteau Laurent, 1989, Mouvements de chômeurs et de précaires en France. La revendication d'un revenu garanti, rapport de recherche pour la Mission interministérielle recherche-expérimentation (MIRE), http://www.cip-idf.org/IMG/pdf/mcp_1989.pdf (consulté le 26 janvier 2017).

BRESSON Yoland, 2007, «Histoire du revenu d'existence », Association pour l'instauration d'un revenu d'existence, http://www.revenudexistence.org/pg/historique.php (consulté le 26 janvier 2017).

Bonnafous Simone, Tournier Maurice, 1995, «Analyse du discours, lexicométrie, communication et politique », Langages, $\mathrm{n}^{\circ} 117$, p. 67-81.

BOUTET Josiane, 2016, Le pouvoir des mots, Paris, La Dispute.

CASTORIADIS Cornélius, 1975, L'institution imaginaire de la société, Paris, Seuil.

ChARAUdeAu Patrick, 2005, Le discours politique : les masques du pouvoir, Paris, Vuibert.

COLIn Nicolas, VERDIER Henri, 2012, L'âge de la multitude : entreprendre et gouverner après la révolution numérique, Paris, Armand Colin.

Cox Robert W., 1981, «Social forces, states and world orders: beyond international relations theory », Millennium: Journal of International Studies, vol. X, n² 2, p. 126-155.

Deroubaix Jean-Claude, Gobin Corinne, 2009, « Mots, fréquence et réseaux dans le discours politique. Analyse lexicométrique, méthode et illustration dans deux corpus de textes européens ", Sociolinguistic Studies, n03, p. 203-227.

Dodeigne Jérémy, Reuchamps Min, VAndeleEne Audrey et al., 2017, «Évaluation de l'allocation universelle. Étude de l'impact des métaphores", La Revue nouvelle, nº 4 , p. 68-77.

FREY Carl Benedikt, OSBORNE Michel A., 2013, «The future of employment: how susceptible are jobs to computerisation?», Oxford, Oxford Martin Programme on Technology and Employment, https://www.oxfordmartin.ox.ac.uk/downloads/academic/ The_Future_of_Employment.pdf (consulté le 26 janvier 2017).

Friedman Milton, 1962, Capitalism and Freedom, Chicago, University of Chicago Press. HeIdEn Serge, MAgUÉ Jean-Philippe, PINCEMIn Bénédicte, 2010, «TXM : une plateforme logicielle open-source pour la textométrie - conception et développement», dans Proceedings of $10^{\text {th }}$ International Conference on the Statistical Analysis of Textual Data (JADT 2010), S. Bolasco, I. Chiari, L. Giuliano éd., Rome, Edizioni Universitarie di Lettere Economia Diritto, vol. II, p. 1021-1032.

HORNY Gérard, 2016, «Le revenu universel, une idée authentiquement libérale », Slate. fr, http://www.slate.fr/story/117269/revenu-universel-progressiste-liberal (consulté le 26 janvier 2016).

LebART Ludovic, SALEm André, 1994, Statistique textuelle, Paris, Dunod.

MÉDA Dominique, 2010, Le travail : une valeur en voie de disparition?, Paris, Flammarion. MOULIER-BOUTANG Yann, 2007, Le capitalisme cognitif: la nouvelle grande transformation, Paris, Éditions Amsterdam.

OIT, 2017, Emploi et questions sociales dans le monde - Tendances 2017, Genève, Organisation internationale du travail (OIT).

SUPLICY Eduardo, 2006, "Citizen's basic income: the answer is blowing in the wind ", USBIG discussion paper n⿳0152, http://www.usbig.net/papers/152-Suplicy-blowin. pdf (consulté le 26 janvier 2017).

VAnderborght Yannick, VAn PARIJS Philippe, 2005, L'allocation universelle, Paris, La Découverte. 


\section{Résumé / Abstract / Compendio}

\section{Le revenu inconditionnel dans et par les discours : analyse lexicométrique et essai de typologie}

Le débat autour du versement d'une forme de revenu inconditionnel connait un succès croissant depuis quelques années en Europe. Ce succès masque toutefois des différences politiques fondamentales entre les propositions en présence que de nombreux partisans du revenu inconditionnel s'emploient à minimiser. Cet article vise au contraire à les mettre en lumière grâce à l'analyse lexicométrique de neuf textes défendant chacun une version particulière de revenu inconditionnel.

Mots-clés : revenu inconditionnel, analyse de discours, lexicométrie

\section{Discourses on unconditional income, towards unconditional income with discourses: lexicometric analysis and proposed typology}

The debate on unconditional forms of income has been increasingly successful in Europe over the last years. That success nevertheless masks fundamental political differences between the various options that are proposed by the numerous promoters of an unconditional income -while the latter try to minimize those differences. This article aims to shed light on those differences with the lexicometric analysis of nine texts which each defend a particularversion of unconditional income.

Keywords: unconditional income, discourse analysis, lexicometric analysis

\section{El ingreso básico incondicional a través de los discursos: análisis lexicomé- trico e intento de tipología}

El debate acerca del pago de una forma de ingreso básico incondicional conoce un éxito creciente en Europa. Sin embargo, este éxito oculta diferencias políticas fundamentales entre las propuestas que numerosos partidarios del ingreso básico incondicional se esfuerzan en minimizar. Al contrario, este artículo apunta develarlas gracias al análisis léxico métrico de nueve textos que defienden, cada uno, una versión particular del ingreso básico incondicional.

Palabras claves: ingreso básico incondicional, análisis del discurso, lexicometría 